\title{
Torsion in the Lichtenbaum Chow group of arithmetic schemes
}

\author{
A. Rosenschon and V. SRinivas
}

\begin{abstract}
We give an example of a smooth arithmetic scheme $\mathfrak{X} \rightarrow B$ over the spectrum of a Dedekind domain and primes $p$ with the property that the $p$-primary torsion subgroup of the Lichtenbaum Chow group $\mathrm{CH}_{\mathrm{L}}^{2}(\mathfrak{X})\{p\}$ has positive corank. This also implies that the unramified cohomology group $\mathrm{H}_{\mathrm{nr}}^{3}\left(\mathfrak{X}, \mathbb{Q}_{p} / \mathbb{Z}_{p}(2)\right)$ is infinite.
\end{abstract}

\section{Introduction}

Let $E$ be an elliptic curve over $\mathbb{Q}$ with conductor $N$ and with complex multiplication by the ring of integers of an imaginary quadratic field $K$, and let $X=E \times_{\mathbb{Q}} E$. Langer-Raskind [15, Theorem 0.1] have shown that if $p$ is a prime not dividing $6 N$, the $p$-primary torsion subgroup $\mathrm{CH}^{2}(X)\{p\}$ of the Chow group of codimension 2 cycles is finite. In fact, they show the following: If $p$ is a prime number not dividing $6 N$, let $B$ be the spectrum of the ring $\mathbb{Z}$ with the primes dividing $6 N p$ inverted, and let $\mathfrak{X}$ be a smooth proper model of $X$ over $B$. By a result of Mildenhall [18, Theorem 5.8] the kernel of the natural map $\mathrm{CH}^{2}(\mathfrak{X}) \rightarrow \mathrm{CH}^{2}(X)$ is finite, which implies that $\mathrm{CH}^{2}(X)\{p\}$ is finite if and only if $\mathrm{CH}^{2}(\mathfrak{X})\{p\}$ is finite. Langer-Raskind use this equivalence and prove the quoted finiteness result for the Chow group of the model.

For both $X$ and $\mathfrak{X}$ (or more generally, smooth schemes over a field or the spectrum of a Dedekind domain) Bloch's cycle complex [2] defines a complex $\mathbb{Z}(n)$ of Zariski sheaves (on the small sites $X_{\text {Zar }}$ or $\mathfrak{X}_{\text {Zar }}$ ), and one can consider the groups

$$
\mathrm{CH}_{\mathrm{M}}^{n}(X)=\mathbb{H}_{\text {Zar }}^{2 n}(X, \mathbb{Z}(n)) \text { and } \operatorname{CH}_{\mathrm{M}}^{n}(\mathfrak{X})=\mathbb{H}_{\text {Zar }}^{2 n}(\mathfrak{X}, \mathbb{Z}(n)) .
$$

It follows from Zariski descent that the group $\mathrm{CH}_{\mathrm{M}}^{n}(X)$ defined in this way coincides with the classical Chow group $\mathrm{CH}^{n}(X)$, and an easy argument shows that in our case of interest we also have $\mathrm{CH}^{2}(\mathfrak{X}) \cong \mathrm{CH}_{\mathrm{M}}^{2}(\mathfrak{X})$. We may also view $\mathbb{Z}(n)$ as a complex of sheaves in the étale topology (on the small 
sites $X_{\text {ét }}$ and $\left.\mathfrak{X}_{\text {ét }}\right)$, and write $\mathbb{Z}(n)$ ét for this complex. The étale motivic or Lichtenbaum Chow groups we consider are the hypercohomology groups

$$
\mathrm{CH}_{\mathrm{L}}^{n}(X)=\mathbb{H}_{\text {ét }}^{2 n}(X, \mathbb{Z}(n)) \quad \text { and } \quad \mathrm{CH}_{\mathrm{L}}^{n}(\mathfrak{X})=\mathbb{H}_{\text {ét }}^{2 n}(\mathfrak{X}, \mathbb{Z}(n)) ;
$$

for details, see Section 2. Since $\mathrm{CH}_{\mathrm{L}}^{2}(\mathfrak{X})\{p\}$ is a quotient and $\mathrm{CH}^{2}(\mathfrak{X})\{p\}$ is a subquotient of $\mathrm{H}_{\text {ét }}^{3}\left(\mathfrak{X}, \mathbb{Q}_{p} / \mathbb{Z}_{p}(2)\right)$, the $p$-primary torsion in both Chow groups is of finite cotype, and a priori there could be more torsion in the Lichtenbaum Chow group. We show:

Theorem 1.1. Let $E / \mathbb{Q}$ be an elliptic curve with complex multiplication and conductor $N$. Let $p$ be a prime such that $p \nmid 6 N$ and such that $E$ has ordinary reduction at $p$, and let $S$ be the set of primes dividing $6 N p$. Set $X=E \times_{\mathbb{Q}} E$, and let $\mathfrak{X} \rightarrow \operatorname{Spec}\left(\mathbb{Z}_{S}\right)$ be a smooth proper model of $X$. Then

(a) $\mathrm{CH}_{\mathrm{L}}^{2}(\mathfrak{X})\{p\}$ has positive corank,

(b) $\mathrm{CH}_{\mathrm{L}}^{2}(X)\{p\}$ contains a copy of $\mathbb{Q}_{p} / \mathbb{Z}_{p}$.

To prove this, we first establish a sufficient criterion in terms of étale cohomology for $\mathrm{CH}_{\mathrm{L}}^{2}(\mathfrak{X})\{p\}$ to have positive corank, and then show that this condition is satisfied. The second step involves local computations using $p$ adic Hodge theory and is already implicit in the proof of Langer-Raskind. For the convenience of the reader we give the full argument.

As suggested by the referee, we state the following corollary. With the notations as in Theorem 1.1, consider the unramified cohomology group

$$
\mathrm{H}_{\mathrm{nr}}^{3}\left(\mathfrak{X}, \mathbb{Q}_{p} / \mathbb{Z}_{p}(2)\right)=\operatorname{ker}\left\{\mathrm{H}_{\text {êt }}^{3}\left(K, \mathbb{Q}_{p} / \mathbb{Z}_{p}(2)\right) \rightarrow \underset{x \in \mathfrak{X}^{(1)}}{\oplus} \mathrm{H}_{\text {êt }}^{2}\left(\kappa(x), \mathbb{Q}_{p} / \mathbb{Z}_{p}(1)\right)\right\},
$$

where $K$ is the function field of $\mathfrak{X}, \mathfrak{X}^{(a)}$ denotes the set of points $x \in \mathfrak{X}$ such that $\operatorname{dim} \mathcal{O}_{X, x}=a$, and $\kappa(x)$ is the residue field of the point.

Corollary 1.2. Let $\mathfrak{X} \rightarrow \operatorname{Spec}\left(\mathbb{Z}_{S}\right)$ and $p$ be as in Theorem 1.1. Then the unramified cohomology group $\mathrm{H}_{\mathrm{nr}}^{3}\left(\mathfrak{X}, \mathbb{Q}_{p} / \mathbb{Z}_{p}(2)\right)$ is infinite. Furthermore, $\mathrm{H}_{\mathrm{nr}}^{3}\left(X, \mathbb{Q}_{p} / \mathbb{Z}_{p}(2)\right)$ is infinite.

\section{Preliminaries}

We summarize the definitions and properties of motivic and Lichtenbaum cohomology needed in the sequel. 
Let $X$ be a smooth quasi-projective variety over a field $k$. The motivic cohomology groups of $X$ with coefficients in an abelian group $A$ are defined as

$$
\mathrm{H}_{\mathrm{M}}^{m}(X, A(n))=\mathbb{H}_{\mathrm{Zar}}^{m}\left(X,\left(z^{n}(-, \bullet) \otimes A\right)[-2 n]\right),
$$

where $z^{n}(-, \bullet)$ is the complex of Zariski sheaves given by Bloch's cycle complex [2]. In particular, $\mathrm{CH}^{n}(X)=\mathrm{H}_{\mathrm{M}}^{2 n}(X, \mathbb{Z}(n))$ is the Chow group of codimension $n$ cycles. The complex $z^{n}(-, \bullet)$ is also a complex of étale sheaves $[2$, $\S 11]$ whose hypercohomology groups define the étale motivic or Lichtenbaum cohomology

$$
\mathrm{H}_{\mathrm{L}}^{m}(X, A(n))=\mathbb{H}_{\text {ét }}^{m}\left(X,\left(z^{n}(-, \bullet)_{\text {ét }} \otimes A\right)[-2 n]\right) ;
$$

in particular, $\mathrm{CH}_{\mathrm{L}}^{n}(X)=\mathrm{H}_{\mathrm{L}}^{2 n}(X, \mathbb{Z}(n))$. It is known that the comparison map

$$
\mathrm{H}_{\mathrm{M}}^{m}(X, A(n)) \rightarrow \mathrm{H}_{\mathrm{L}}^{m}(X, A(n))
$$

is an isomorphism with rational coefficients. Furthermore, if $\ell \nmid \operatorname{char}(k)$ is a prime, there is a quasi-isomorphism $\left(\mathbb{Z} / \ell^{r} \mathbb{Z}\right)(n)_{\text {ét }} \stackrel{\sim}{\rightarrow} \mu_{\ell^{r}}^{\otimes n}[10$, Theorem 1.5], thus with finite coefficients Lichtenbaum and étale cohomology coincides

$$
\mathrm{H}_{\mathrm{L}}^{m}\left(X, \mathbb{Z} / \ell^{r}(n)\right) \cong \mathrm{H}_{\text {ét }}^{m}\left(X, \mu_{\ell^{r}}^{\otimes n}\right) .
$$

Let $R$ be a Dedekind domain and let $\mathfrak{X} \rightarrow B=\operatorname{Spec}(R)$ be an essentially smooth $B$-scheme. The complex $z^{n}(-, \bullet)$ on $\mathfrak{X}$ also defines a complex of sheaves for the Zariski and for the étale cohomology. Following Geisser [11], we define the motivic and étale motivic or Lichtenbaum cohomology of $\mathfrak{X}$ as

$$
\mathrm{H}_{\mathrm{M}}^{m}(\mathfrak{X}, A(n))=\mathbb{H}_{\mathrm{Zar}}^{m}(\mathfrak{X}, A(n)) \quad \text { and } \quad \mathrm{H}_{\mathrm{L}}^{m}(\mathfrak{X}, A(n))=\mathbb{H}_{\text {ét }}^{m}(\mathfrak{X}, A(n)) \text {; }
$$

we also set $\mathrm{CH}_{\mathrm{M}}^{n}(\mathfrak{X})=\mathrm{H}_{\mathrm{M}}^{2 n}(\mathfrak{X}, \mathbb{Z}(n))$ and $\mathrm{CH}_{\mathrm{L}}^{n}(\mathfrak{X})=\mathrm{H}_{\mathrm{L}}^{2 n}(\mathfrak{X}, \mathbb{Z}(n))$. We remark that it not clear that the groups $\mathrm{H}_{\mathrm{M}}^{m}(\mathfrak{X}, \mathbb{Z}(n))$ coincides with the cohomology of Bloch's cycle complex of abelian groups in general (this is known, for example, in case $X$ is essentially of finite type over $B=\operatorname{Spec}(A)$, where $A$ is a discrete valuation ring [11, Theorem 3.2]). However, in our case of interest we can compare the exact localization sequences along the evident maps

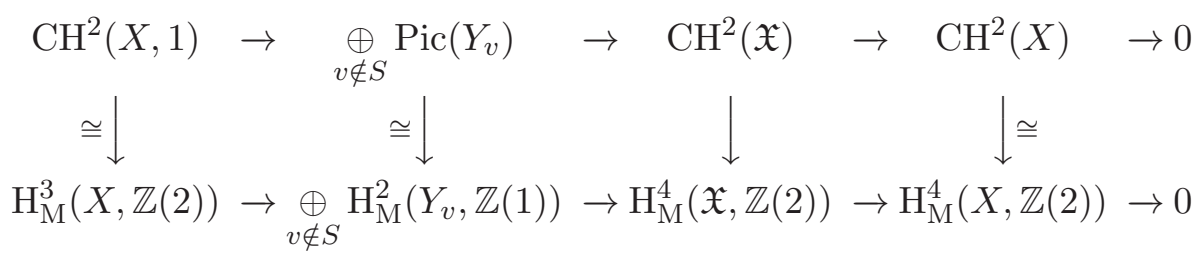


where the isomorphisms result from the facts that for $X$ one has Zariski descent, and that for a smooth variety $Y$ one has $\mathbb{Z}(1)_{\text {Zar }} \sim \mathcal{O}_{Y}^{\times}[-1][2$, Corollary 6.4]. Hence $\mathrm{CH}^{2}(\mathfrak{X}) \cong \mathrm{CH}_{\mathrm{M}}^{2}(\mathfrak{X})$, i.e. the motivic Chow group of the model we consider does indeed coincide with the classical Chow group.

We remark that if $\epsilon: \mathfrak{X}_{\text {ét }} \rightarrow \mathfrak{X}_{\text {Zar }}$ is the canonical morphism of sites and $\mathfrak{X}$ is essentially of finite type over $B$, the map $\mathbb{Q}(n)_{\text {Zar }} \rightarrow R \epsilon_{*} \mathbb{Q}(n)_{\text {ét }}$ is a quasiisomorphism [11, Proposition 3.6], hence we have with rational coefficients

$$
\mathrm{H}_{\mathrm{M}}^{m}(\mathfrak{X}, \mathbb{Q}(n)) \cong \mathrm{H}_{\mathrm{L}}^{m}(\mathfrak{X}, \mathbb{Q}(n)) .
$$

Let $\mathfrak{X} \rightarrow B$ be an essentially smooth scheme over the spectrum of a Dedekind ring. In what follows we will use two properties of the Lichtenbaum cohomology groups of $\mathfrak{X}$ whose proofs require the use of the proof of the Bloch-Kato conjecture, i.e. the assertion that for a field $F$ and a prime number $p \nmid \operatorname{char}(F)$ the norm-residue map between Milnor $K$-theory and étale cohomology

$$
\mathrm{K}_{m}^{M}(F) / p^{r} \rightarrow \mathrm{H}_{\mathrm{et}}^{m}\left(F, \mu_{p^{r}}^{\otimes m}\right)
$$

is an isomorphism. This has been shown for the prime $p=2$ by Voevodsky [23], and in general by Rost-Voevodsky [24], see also [25]. Making use of the norm-residue isomorphism, Geisser has shown the following [11, Theorem 1.2]:

(A) The morphism $\mathbb{Z}(n)_{\mathrm{Zar}} \rightarrow \tau_{\leq n+1} R \epsilon_{*} \mathbb{Z}(n)_{\text {ét }}$ is a quasi-isomorphism, thus

$$
\mathrm{H}_{\mathrm{M}}^{m}(\mathfrak{X}, \mathbb{Z}(n)) \cong \mathrm{H}_{\mathrm{L}}^{m}(\mathfrak{X}, \mathbb{Z}(n)) \text { for } m \leq n+1 .
$$

(B) If the prime $p$ is invertible in $B$, there is a quasi-isomorphism of complexes of étale sheaves $\mathbb{Z} / p^{r}(n)_{\text {ét }} \stackrel{\sim}{\rightarrow} \mu_{p^{r}}^{\otimes n}$ (where the sheaf $\mu_{p^{r}}^{\otimes n}$ is in degree 0). Hence

$$
\mathrm{H}_{\mathrm{L}}^{m}\left(\mathfrak{X}, \mathbb{Z} / p^{r}(n)\right) \cong \mathrm{H}_{\text {ét }}^{m}\left(\mathfrak{X}, \mu_{p^{r}}^{\otimes n}\right) \text { for } m \in \mathbb{Z} .
$$

\section{Proof of the Theorem}

Proof. (of Theorem 1.1) Let $p>3$ be a prime such that $E$ has good ordinary reduction at $p$, and let $X=E \times \mathbb{Q} E$ and $\mathfrak{X} \rightarrow \operatorname{Spec}\left(\mathbb{Z}_{S}\right)$ be as in Theorem 1.1; in particular, the prime $p$ is invertible in $\mathbb{Z}_{S}$. Note that by standard arguments involving the Leray spectral sequence associated with the morphism $\mathfrak{X} \rightarrow \operatorname{Spec}\left(\mathbb{Z}_{S}\right)$, the étale cohomology groups $H_{\text {ét }}^{m}\left(\mathfrak{X}, \mu_{p^{n}}^{\otimes n}\right)$ are finite, the groups $\mathrm{H}_{\mathrm{ét}}^{m}\left(\mathfrak{X}, \mathbb{Q}_{p}(n)\right)$ are finite-dimensional $\mathbb{Q}_{p}$-vector spaces, and the 
groups $\mathrm{H}_{\text {ét }}^{m}\left(\mathfrak{X}, \mathbb{Q}_{p} / \mathbb{Z}_{p}(n)\right)$ are of finite cotype, see [15, proof of Lemma 3.1], for instance.

In both topologies $\tau=$ Zar and $\tau=$ ét, the map 'multiplication by $p^{r}$ ' induces the short exact sequence $0 \rightarrow \mathbb{Z}(2)_{\tau} \rightarrow \mathbb{Z}(2)_{\tau} \rightarrow \mathbb{Z} / p^{r}(2)_{\tau} \rightarrow 0$ of complexes of sheaves, from which we obtain short exact universal coefficient sequences in motivic and Lichtenbaum cohomology. Comparing these sequences along the morphism $\mathfrak{X}_{\text {ét }} \rightarrow \mathfrak{X}_{\text {Zar }}$, we get the following commutative diagram with exact rows

$$
\begin{aligned}
& 0 \rightarrow \mathrm{H}_{\mathrm{M}}^{3}(\mathfrak{X}, \mathbb{Z}(2)) / p^{r} \rightarrow \mathrm{H}_{\mathrm{M}}^{3}\left(\mathfrak{X}, \mathbb{Z} / p^{r}(2)\right) \rightarrow \mathrm{CH}^{2}(\mathfrak{X})\left[p^{r}\right] \rightarrow 0 \\
& 0 \rightarrow \mathrm{H}_{\mathrm{L}}^{3}(\mathfrak{X}, \mathbb{Z}(2)) / p^{r} \rightarrow \mathrm{H}_{\text {ét }}^{3}\left(\mathfrak{X}, \mu_{p^{r}}^{\otimes 2}\right) \quad \rightarrow \mathrm{CH}_{\mathrm{L}}^{2}(\mathfrak{X})\left[p^{r}\right] \rightarrow 0
\end{aligned}
$$

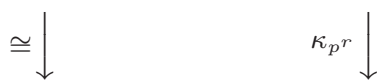

Here we have used the quasi-isomorphism $\mathbb{Z}(n) \stackrel{\sim}{\rightarrow} \tau_{\leq n+1} R \epsilon_{*} \mathbb{Z}(n)_{\text {ét }}$ from (A) to obtain the left vertical isomorphism and the quasi-isomorphism $\mathbb{Z} / p^{r}(2)$ ét $\stackrel{\sim}{\rightarrow} \mu_{p^{r}}^{\otimes 2}$ from $(\mathrm{B})$, which allows us to identify in the bottom row the groups

$$
\mathrm{H}_{\mathrm{L}}^{3}\left(\mathfrak{X}, \mathbb{Z} / p^{r}(2)\right) \cong \mathrm{H}_{\text {ét }}^{3}\left(\mathfrak{X}, \mu_{p^{r}}^{\otimes 2}\right)
$$

Note that, as remarked above, $\mathrm{H}_{\text {ét }}^{3}\left(\mathfrak{X}, \mu_{p^{r}}^{\otimes 2}\right)$ is finite. Furthermore, the kernel of the map $\mathrm{CH}^{2}(\mathfrak{X}) \rightarrow \mathrm{CH}^{2}(X)$ is finite $\left[18\right.$, Theorem 5.8] and $\mathrm{CH}^{2}(X)\left[p^{r}\right]$ is finite $[15$, Theorem 0.1$]$, thus $\mathrm{CH}^{2}(\mathfrak{X})\left[p^{r}\right]$ is finite, and hence all groups in the above diagram are finite as well. Taking the direct limit over all $r$ of the bottom row shows that $\mathrm{CH}_{\mathrm{L}}^{2}(\mathfrak{X})\{p\}$ is a quotient of $\mathrm{H}_{\text {ét }}^{3}\left(\mathfrak{X}, \mathbb{Q}_{p} / \mathbb{Z}_{p}(2)\right)$, thus is itself of finite cotype. Hence we have an isomorphism

$$
\mathrm{CH}_{\mathrm{L}}^{2}(\mathfrak{X})\{p\} \cong\left(\mathbb{Q}_{p} / \mathbb{Z}_{p}\right)^{\oplus k} \oplus F, \quad F \text { a finite group. }
$$

If $A$ is an abelian group, let $\mathrm{T}_{p}(A)=\operatorname{Hom}\left(\mathbb{Q}_{p} / \mathbb{Z}_{p}, A\right)$ be the $p$-adic Tate module, and let $V_{p}(A)=\mathrm{T}_{p}(A) \otimes_{\mathbb{Z}_{p}} \mathbb{Q}_{p}$ be the corresponding Tate $\mathbb{Q}_{p}$-vector space. From the above description of $\mathrm{CH}_{\mathrm{L}}^{2}(\mathfrak{X})\{p\}$ we see that $\mathrm{T}_{p}\left(\mathrm{CH}_{\mathrm{L}}^{2}(\mathfrak{X})\{p\}\right) \cong \mathbb{Z}_{p}^{\oplus k}$, thus $\mathrm{V}_{p}\left(\mathrm{CH}_{\mathrm{L}}^{2}(\mathfrak{X})\{p\}\right) \cong \mathbb{Q}_{p}^{\oplus k}$, and we have

$$
\mathrm{CH}_{\mathrm{L}}^{2}(\mathfrak{X})\{p\} \text { has positive corank } \Leftrightarrow V_{p}\left(\mathrm{CH}_{\mathrm{L}}^{2}(\mathfrak{X})\{p\}\right) \neq 0 .
$$

Consider the $\mathbb{Q}_{p}$-vector spaces

$$
\begin{aligned}
\mathrm{H}_{\mathrm{M}}(\mathfrak{X}) & =\left[\underset{\leftarrow}{\left[\lim _{r}\right.} \mathrm{H}_{\mathrm{M}}^{3}(\mathfrak{X}, \mathbb{Z}(2)) / p^{r}\right] \otimes_{\mathbb{Z}_{p}} \mathbb{Q}_{p}, \\
\mathrm{H}_{\mathrm{L}}(\mathfrak{X}) & =\left[\underset{\leftarrow}{\lim _{\leftarrow}} \mathrm{H}_{\mathrm{L}}^{3}(\mathfrak{X}, \mathbb{Z}(2)) / p^{r}\right] \otimes_{\mathbb{Z}_{p}} \mathbb{Q}_{p} .
\end{aligned}
$$


Since $\mathrm{CH}^{2}(\mathfrak{X})\{p\}$ is finite, $\mathrm{T}_{p}\left(\mathrm{CH}^{2}(\mathfrak{X})\{p\}\right)=0$ and $\mathrm{V}_{p}\left(\mathrm{CH}^{2}(\mathfrak{X})\{p\}\right)=0$. Taking in (1) the inverse limit over all $r$ (which is exact in this case) and tensoring with $\mathbb{Q}_{p}$, we obtain the following commutative diagram whose bottom row is exact

$$
\begin{aligned}
& \mathrm{H}_{\mathrm{M}}(\mathfrak{X}) \stackrel{\cong}{\rightrightarrows}\left[\lim _{\leftarrow} \mathrm{H}_{\mathrm{M}}^{3}\left(\mathfrak{X}, \mu_{p^{r}}^{\otimes 2}\right)\right] \otimes_{\mathbb{Z}_{p}} \mathbb{Q}_{p} \\
& \underset{\downarrow}{\cong} \underset{\mathrm{H}_{\mathrm{L}}(\mathfrak{X})}{\rightarrow} \quad \stackrel{\kappa \downarrow}{\mathrm{H}_{\text {ét }}^{3}\left(\mathfrak{X}, \mathbb{Q}_{p}(2)\right)} \rightarrow \mathrm{V}_{p}\left(\mathrm{CH}_{\mathrm{L}}^{2}(\mathfrak{X})\{p\}\right) \rightarrow 0
\end{aligned}
$$

If $\mathbb{K}=\operatorname{im}(\kappa)$, this immediately implies that

$$
\mathrm{V}_{p}\left(\mathrm{CH}_{\mathrm{L}}^{2}(\mathfrak{X})\{p\}\right) \cong \mathrm{H}_{\text {ét }}^{3}\left(\mathfrak{X}, \mathbb{Q}_{p}(2)\right) / \mathbb{K} .
$$

If $\mathbb{Q}(X)$ is the function field of $X$, define

$$
\mathrm{NH}_{\text {ét }}^{3}\left(\mathfrak{X}, \mu_{p^{r}}^{\otimes 2}\right)=\operatorname{ker}\left\{\mathrm{H}_{\text {ét }}^{3}\left(\mathfrak{X}, \mu_{p^{r}}^{\otimes 2}\right) \rightarrow \mathrm{H}^{3}\left(\mathbb{Q}(X), \mu_{p^{r}}^{\otimes 2}\right)\right\},
$$

and

$$
\mathrm{NH}^{3}\left(\mathfrak{X}, \mathbb{Q}_{p}(2)\right)=\left[\lim _{r} \mathrm{~N} \mathrm{H}_{\text {ét }}^{3}\left(\mathfrak{X}, \mu_{p^{r}}^{\otimes 2}\right)\right] \otimes_{\mathbb{Z}_{p}} \mathbb{Q}_{p} .
$$

Note that the $\mathbb{Q}_{p}$-vector space $\mathrm{NH}_{\text {ét }}^{3}\left(\mathfrak{X}, \mathbb{Q}_{p}(2)\right)$ defined above does not necessarily coincide with the first level of the usual coniveau filtration, i.e. if

$$
\mathrm{N}^{\prime} \mathrm{H}_{\text {ét }}^{3}\left(\mathfrak{X}, \mathbb{Q}_{p}(2)\right)=\lim _{\rightarrow} \operatorname{ker}\left\{\mathrm{H}_{\text {ét }}^{3}\left(\mathfrak{X}, \mathbb{Q}_{p}(2)\right) \rightarrow \mathrm{H}_{\text {êt }}^{3}\left(\mathfrak{X} \backslash \mathfrak{Z}, \mathbb{Q}_{p}(2)\right)\right\},
$$

where the limit is taken over all closed subschemes $\mathfrak{Z} \subset \mathfrak{X}$ of codimension one, there is an injective map $\mathrm{N}^{\prime} \mathrm{H}_{\text {ét }}^{3}\left(\mathfrak{X}, \mathbb{Q}_{p}(2)\right) \rightarrow \mathrm{NH}_{\text {ét }}^{3}\left(\mathfrak{X}, \mathbb{Q}_{p}(2)\right)$, which is not an isomorphism in general, see [15, Remark 2.1].

We claim that $\mathbb{K}=\operatorname{im}(\kappa) \subseteq \mathrm{NH}_{\text {ét }}^{3}\left(\mathfrak{X}, \mathbb{Q}_{p}(2)\right)$. To see this, note first that

$$
\left.\mathbb{K}=\underset{r}{\lim } \operatorname{im}\left(\kappa_{p^{r}}\right)\right] \otimes_{\mathbb{Z}_{p}} \mathbb{Q}_{p}
$$

The composition $\mathrm{H}_{\mathrm{M}}^{3}\left(\mathfrak{X}, \mathbb{Z} / p^{r}(2)\right) \stackrel{\kappa_{p^{r}}}{\rightarrow} \mathrm{H}_{\text {ét }}^{3}\left(\mathfrak{X}, \mu_{p^{r}}^{\otimes 2}\right) \rightarrow \mathrm{H}_{\text {ét }}^{3}\left(\mathbb{Q}(X), \mu_{p^{r}}^{\otimes 2}\right)$ coincides with $\mathrm{H}_{\mathrm{M}}^{3}\left(\mathfrak{X}, \mathbb{Z} / p^{r}(2)\right) \rightarrow \mathrm{H}_{\mathrm{M}}^{3}\left(\mathbb{Q}(X), \mathbb{Z} / p^{r}(2)\right) \rightarrow \mathrm{H}_{\text {ét }}^{3}\left(\mathbb{Q}(X), \mathbb{Z} / p^{r}(2)\right)$, where the first map is the zero map, because the motivic Zariski sheaf $\mathcal{H}_{M}^{3}\left(\mathbb{Z} / p^{r}(2)\right)$ on $X$ is trivial (this follows from the existence of a Gersten resolution $[2, \S 10]$ and a local argument; see also [11, §4]). Thus $\operatorname{im}\left(\kappa_{p^{r}}\right) \subseteq$ $\mathrm{NH}_{\text {ét }}^{3}\left(\mathfrak{X}, \mu_{p^{r}}^{\otimes 2}\right)$, and our claim follows from (4). 
Given $\mathbb{K} \subseteq \mathrm{N} \mathrm{H}_{\text {ét }}^{3}\left(\mathfrak{X}, \mathbb{Q}_{p}(2)\right)$, the composition of (3) with the evident quotient $\operatorname{map} \mathrm{H}_{\text {ét }}^{3}\left(\mathfrak{X}, \mathbb{Q}_{p}(2)\right) / \mathbb{K} \rightarrow \mathrm{H}_{\text {ét }}^{3}\left(\mathfrak{X}, \mathbb{Q}_{p}(2)\right) / \mathrm{NH}_{\text {ét }}^{3}\left(\mathfrak{X}, \mathbb{Q}_{p}(2)\right)$ yields a surjective map

$$
\mathrm{V}_{p}\left(\mathrm{CH}_{\mathrm{L}}^{2}(\mathfrak{X})\{p\}\right) \rightarrow \mathrm{H}_{\text {ét }}^{3}\left(\mathfrak{X}, \mathbb{Q}_{p}(2)\right) / \mathrm{NH}_{\text {ét }}^{3}\left(\mathfrak{X}, \mathbb{Q}_{p}(2)\right) .
$$

In particular, to show $\mathrm{CH}_{\mathrm{L}}^{2}(\mathfrak{X})\{p\}$ has positive corank it suffices to show

$$
\operatorname{dim}_{\mathbb{Q}_{p}} \mathrm{NH}_{\text {ét }}^{3}\left(\mathfrak{X}, \mathbb{Q}_{p}(2)\right) \leq \operatorname{dim}_{\mathbb{Q}_{p}} \mathrm{H}_{\text {ét }}^{3}\left(\mathfrak{X}, \mathbb{Q}_{p}(2)\right)-1 \text {. }
$$

Let $G_{S}$ be the Galois group of the maximal extension of $\mathbb{Q}$ in $\overline{\mathbb{Q}}$ which is unramified outside of $S$. For $v \in S$ let $X_{v}=\mathfrak{X} \times{ }_{B} \mathbb{Q}_{v}$ and write $G_{v}=$ $\operatorname{Gal}\left(\overline{\mathbb{Q}}_{v} / \mathbb{Q}_{v}\right)$ for the absolute Galois group and $\mathrm{H}^{n}\left(G_{v}, M\right)=\mathrm{H}^{n}\left(\mathbb{Q}_{v}, M\right)$ for the continuous Galois cohomology groups with values in a $G_{v}$-module $M$.

Let $V=\mathrm{H}_{\text {ét }}^{2}\left(\bar{X}, \mathbb{Q}_{p}(2)\right)$ and consider the localization map

$$
\mathrm{H}^{1}\left(G_{S}, V\right) \rightarrow \underset{v \in S}{\oplus} \mathrm{H}^{1}\left(G_{v}, V\right)
$$

we recall that by results of Flach [7] this map becomes surjective after passing to a suitable quotient on the right hand side.

In general, given a $\mathbb{Q}_{p}$-linear representation $V$ of $G_{\mathbb{Q}}$, we have for a nonarchimedian completion $\mathbb{Q}_{v}$ of $\mathbb{Q}$ the following $\mathbb{Q}_{p}$-subspaces of $\mathrm{H}^{1}\left(\mathbb{Q}_{v}, V\right)$

$$
\mathrm{H}_{f}^{1}\left(G_{v}, V\right) \subseteq \mathrm{H}_{g}^{1}\left(G_{v}, V\right) \subseteq \mathrm{H}^{1}\left(G_{v}, V\right)
$$

defined by Bloch-Kato in $[3, \S 3]$ as follows: If $v \neq p$, let $\mathbb{Q}_{v}^{n r}$ be the maximal unramified extension of $\mathbb{Q}_{v}$, set $\mathrm{H}_{g}^{1}\left(\mathbb{Q}_{v}, V\right)=\mathrm{H}^{1}\left(\mathbb{Q}_{v}, V\right)$, and

$$
\mathrm{H}_{f}^{1}\left(\mathbb{Q}_{v}, V\right)=\operatorname{ker}\left\{\mathrm{H}^{1}\left(\mathbb{Q}_{v}, V\right) \rightarrow \mathrm{H}^{1}\left(\mathbb{Q}_{v}^{n r}, V\right)\right\} .
$$

If $v=p$, let $B_{c r i s}$ and $B_{D R}$ be the rings defined by Fontaine in [8], and set

$$
\begin{aligned}
& \mathrm{H}_{f}^{1}\left(\mathbb{Q}_{v}, V\right)=\operatorname{ker}\left\{\mathrm{H}^{1}\left(\mathbb{Q}_{v}, V\right) \rightarrow \mathrm{H}^{1}\left(\mathbb{Q}_{v}, V \otimes_{\mathbb{Q}_{p}} B_{c r i s}\right)\right\}, \\
& \mathrm{H}_{g}^{1}\left(\mathbb{Q}_{v}, V\right)=\operatorname{ker}\left\{\mathrm{H}^{1}\left(\mathbb{Q}_{v}, V\right) \rightarrow \mathrm{H}^{1}\left(\mathbb{Q}_{v}, V \otimes_{\mathbb{Q}_{p}} B_{D R}\right)\right\} .
\end{aligned}
$$

If $T$ is a $G_{S}$-stable $\mathbb{Z}_{p}$-lattice, define $A$ by the exactness of the sequence

$$
0 \rightarrow T \stackrel{\iota}{\rightarrow} V \stackrel{\text { pr }}{\rightarrow} A \rightarrow 0 .
$$

Let $W_{v}=\mathrm{H}_{f}^{1}\left(\mathbb{Q}_{v}, V\right) \subseteq \mathrm{H}^{1}\left(\mathbb{Q}_{v}, V\right)$ (where we set $\mathrm{H}_{f}^{1}\left(\mathbb{Q}_{v}, V\right)=0$ for the archimedian place $v=\infty)$. Let $\mathrm{M}_{\mathbb{Q}}$ be the set of all valuations of $\mathbb{Q}$ and 
define

$$
\mathrm{H}_{f, \mathbb{Z}}^{1}(\mathbb{Q}, V)=\left\{x \in \mathrm{H}^{1}(\mathbb{Q}, V) \mid x_{v} \in \mathrm{H}_{f}^{1}\left(\mathbb{Q}_{v}, V\right) \text { for all } v \in \mathrm{M}_{\mathbb{Q}}\right\} .
$$

Using Tate's global duality theorem, Flach has shown [7, Proposition 1.4] (cp. [3, Lemma 5.16]) that the localization maps induce an exact sequence

$$
\mathrm{H}^{1}\left(G_{S}, A\right) \rightarrow \underset{v \in S}{\oplus} \frac{\mathrm{H}^{1}\left(\mathbb{Q}_{v}, A\right)}{\operatorname{pr}_{*}\left(W_{v}\right)} \rightarrow \iota_{*}^{-1}\left(W^{\prime}\right)^{*},
$$

where

$$
W^{\prime}=\mathrm{H}_{f, \mathbb{Z}}^{1}\left(\mathbb{Q}, V^{*}(1)\right) \text { and } V^{*}(1) \cong \mathrm{H}_{\text {êt }}^{2}\left(\bar{X}, \mathbb{Q}_{p}(1)\right) .
$$

Flach also showed that given our assumptions on $E$ the Selmer groups associated with $V$ and $V^{*}(1)$ are finite over $\mathbb{Q}$ [7]. This implies that the group $W^{\prime}$ appearing in the above exact sequence is finite, and further that for the $\mathbb{Q}_{p}$-vector space $V=\mathrm{H}_{\text {êt }}^{2}\left(\bar{X}, \mathbb{Q}_{p}(2)\right)$ the localization maps induce a surjective map [15, Corollary 5.4]

$$
\mathrm{H}^{1}\left(G_{S}, V\right) \rightarrow \underset{v \in S}{\oplus} \frac{\mathrm{H}^{1}\left(\mathbb{Q}_{v}, V\right)}{\mathrm{H}_{f}^{1}\left(\mathbb{Q}_{v}, \mathrm{~V}\right)} .
$$

Consider now a smooth proper model $\mathfrak{X} \rightarrow \operatorname{Spec}\left(\mathbb{Z}_{S}\right)$ of $X$ as in Theorem 1.1. The surjective localization map from (7) fits into the commutative diagram

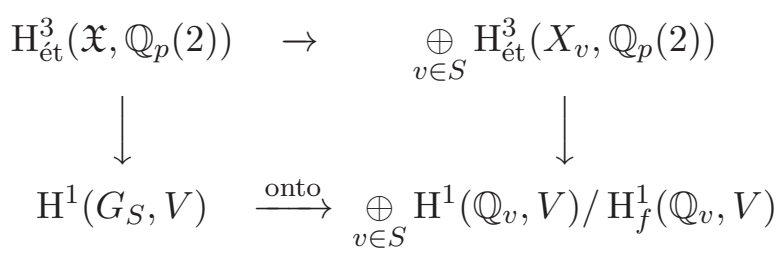

where the left vertical map is induced by the Hochschild-Serre spectral sequence, and the right vertical map comes from the Hochschild-Serre spectral sequence, followed by the evident quotient map. We claim the left vertical map $\mathrm{H}_{\text {ét }}^{3}\left(\mathfrak{X}, \mathbb{Q}_{p}(2)\right) \rightarrow \mathrm{H}^{1}\left(G_{S}, V\right)$ is surjective. Indeed, $\mathrm{E}_{2}^{r, s}=\mathrm{H}^{r}\left(G_{S}\right.$, $\left.\mathrm{H}^{s}\left(\bar{X}, \mathbb{Q}_{p}(2)\right)\right)=0$ for $r>2$ by cohomological dimension [4, 2.11], and $\mathrm{E}_{2}^{0,3}=0$ by a standard specialization argument, see [5, Theorem 1.5]. Thus $\operatorname{gr}_{F}^{0} \mathrm{H}_{\text {ét }}^{3}\left(\mathfrak{X}, \mathbb{Q}_{p}(2)\right)=0$ and $\mathrm{E}_{2}^{1,2}=\operatorname{gr}_{F}^{1} \mathrm{H}_{\text {ét }}^{3}\left(\mathfrak{X}, \mathbb{Q}_{p}(2)\right)$, i.e. the claimed surjection is the evident map $\mathrm{F}^{1} \mathrm{H}_{\text {êt }}^{3}\left(\mathfrak{X}, \mathbb{Q}_{p}(2)\right) \rightarrow \operatorname{gr}_{F}^{1} \mathrm{H}_{\text {ét }}^{3}\left(\mathfrak{X}, \mathbb{Q}_{p}(2)\right)$. Since $p \in S$, we may pass first to the quotient $\mathrm{H}^{1}\left(\mathbb{Q}_{p}, V\right) / \mathrm{H}_{f}^{1}\left(\mathbb{Q}_{p}, V\right)$, and then further 
to $\mathrm{H}^{1}\left(\mathbb{Q}_{p}, V\right) / \mathrm{H}_{g}^{1}\left(\mathbb{Q}_{p}, V\right)$ to obtain a surjective map of $\mathbb{Q}_{p}$-vector spaces

$$
\mathrm{H}_{\text {ét }}^{3}\left(\mathfrak{X}, \mathbb{Q}_{p}(2)\right) \rightarrow \mathrm{H}^{1}\left(\mathbb{Q}_{p}, V\right) / \mathrm{H}_{g}^{1}\left(\mathbb{Q}_{p}, V\right) .
$$

In our next step we show that the surjective map (8) factors through the quotient $\mathrm{H}_{\text {ét }}^{3}\left(\mathfrak{X}, \mathbb{Q}_{p}(2)\right) / \mathrm{N} \mathrm{H}_{\text {ét }}^{3}\left(\mathfrak{X}, \mathbb{Q}_{p}(2)\right)$. In the Hochschild-Serre spectral sequence

$$
\mathrm{E}_{2}^{r, s}=\mathrm{H}^{r}\left(\mathbb{Q}_{p}, \mathrm{H}_{\text {ét }}^{r}\left(\bar{X}, \mathbb{Q}_{p}(2)\right)\right) \Rightarrow \mathrm{H}_{\text {ét }}^{r+s}\left(X_{\mathbb{Q}_{p}}, \mathbb{Q}_{p}(2)\right)
$$

$\mathrm{E}_{2}^{0,3}=0$ by a weight argument, and $\mathrm{E}_{2}^{2,1}=0$ by [1, Proposition 2.4]. Since furthermore $\mathrm{E}_{2}^{r, s}=0$ for $r>2$ by cohomological dimension, this implies that

$$
\mathrm{H}_{\text {ét }}^{3}\left(X_{\mathbb{Q}_{p}}, \mathbb{Q}_{p}(2)\right) \cong \mathrm{H}^{1}\left(\mathbb{Q}_{p}, V\right) \text {. }
$$

Let $\mathfrak{X}_{\mathbb{Z}_{p}}=\mathfrak{X} \times_{B} \mathbb{Z}_{p}$, and let $j: X_{\mathbb{Q}_{p}} \rightarrow \mathfrak{X}_{\mathbb{Z}_{p}}$ be the inclusion. It is immediate that the image of $\mathrm{NH}_{\text {ét }}^{3}\left(\mathfrak{X}, \mathbb{Q}_{p}(2)\right)$ in $\mathrm{H}^{1}\left(\mathbb{Q}_{p}, V\right)$ is contained in the image of $\mathrm{NH}_{\text {ét }}^{3}\left(\mathfrak{X}_{\mathbb{Z}_{p}}, \mathbb{Q}_{p}(2)\right)$ in $\mathrm{H}^{1}\left(\mathbb{Q}_{p}, V\right)$. In particular, to show the map (8) factors through $\mathrm{H}_{\text {ét }}^{3}\left(\mathfrak{X}, \mathbb{Q}_{p}(2)\right) / \mathrm{NH}_{\text {ét }}^{3}\left(\mathfrak{X}, \mathbb{Q}_{p}(2)\right)$ it suffices to show that the image of $\mathrm{NH}_{\text {ét }}^{3}\left(\mathfrak{X}_{\mathbb{Z}_{p}}, \mathbb{Q}_{p}(2)\right)$ in $\mathrm{H}^{1}\left(\mathbb{Q}_{p}, V\right)$ is contained in the subspace $\mathrm{H}_{g}^{1}\left(\mathbb{Q}_{p}, V\right)$.

If $C^{\bullet}$ is a complex of sheaves on $\mathfrak{X}_{\mathbb{Z}_{p}}$, we write $\tau_{\leq n} C^{\bullet}$ for its truncation consisting of terms in degree $\leq n$. In particular, if $C^{\bullet}=R j_{*} \mu_{p^{r}}^{\otimes 2}$, then

$$
\mathrm{H}^{3}\left(\mathfrak{X}_{\mathbb{Z}_{p}}, \tau_{\leq 2} R j_{*} \mathbb{Q}_{p}(2)\right)=\left[\underset{r}{\left[\lim _{r}\right.} \mathrm{H}^{3}\left(\mathfrak{X}_{\mathbb{Z}_{p}}, \tau_{\leq 2} R j_{*} \mu_{p^{r}}^{\otimes 2}\right)\right] \otimes_{\mathbb{Z}_{p}} \mathbb{Q}_{p}
$$

and it follows from [16, Lemma 5-4] that there is an inclusion

$$
\mathrm{NH}_{\text {ét }}^{3}\left(\mathfrak{X}_{\mathbb{Z}_{p}}, \mathbb{Q}_{p}(2)\right) \subseteq \mathrm{H}^{3}\left(\mathfrak{X}_{\mathbb{Z}_{p}}, \tau_{\leq 2} R j_{*} \mathbb{Q}_{p}(2)\right) .
$$

We need a result from $p$-adic Hodge theory, for an overview in a more general setting, see $[20, \S 2]$, for instance. There is a natural (injective) pullback map

$$
\alpha: \mathrm{H}^{3}\left(\mathfrak{X}_{\mathbb{Z}_{p}}, \tau_{\leq 2} R j_{*} \mathbb{Q}_{p}(2)\right) \rightarrow \mathrm{H}_{\text {ét }}^{3}\left(X_{\mathbb{Q}_{p}}, \mathbb{Q}_{p}(2)\right) \cong \mathrm{H}^{1}\left(\mathbb{Q}_{p}, V\right) .
$$

Let $\iota: Y_{p} \rightarrow \mathfrak{X}_{\mathbb{Z}_{p}}$ be the inclusion of the closed fiber, and let $s_{r} \log _{(n)}$ be the $\log$ syntomic complex defined by Kato in [14]. By a result of Tsuji [22, Theorem 5.1], we have in $D_{\text {ét }}^{b}\left(\mathfrak{X}, \mathbb{Z} / p^{r} \mathbb{Z}\right)$ for $0 \leq n \leq p-2$ a quasi-isomorphism

$$
\eta: s_{r}^{\log }(n) \stackrel{\sim}{\rightarrow} \iota_{*} \iota^{*}\left(\tau_{\leq n} R j_{*} \mu_{p^{r}}^{\otimes n}\right)
$$


in particular, this holds for $n=2$, provided $p>3$. Consider now the group

$$
\mathrm{H}^{n}\left(\mathfrak{X}_{\mathbb{Z}_{p}}, s_{\mathbb{Q}_{p}}^{\log }(n)\right)=\left[\underset{r}{\lim _{\leftarrow}} \mathrm{H}^{n}\left(\mathfrak{X}_{\mathbb{Z}_{p}}, s_{r}^{\log }(n)\right)\right] \otimes_{\mathbb{Z}_{p}} \mathbb{Q}_{p}
$$

Since we assume $p>3$, the quasi-isomorphism $\eta$ induces an isomorphism

$$
\mathrm{H}^{3}\left(\mathfrak{X}_{\mathbb{Z}_{p}}, s_{\mathbb{Q}_{p}}^{\log }(2)\right) \stackrel{\cong}{\rightrightarrows} \mathrm{H}^{3}\left(\mathfrak{X}_{\mathbb{Z}_{p}}, \tau_{\leq 2} R j_{*} \mathbb{Q}_{p}(2)\right) .
$$

Let $\eta^{\prime}$ be the composition of this isomorphism with the map $\alpha$ from (11), i.e.

$$
\eta^{\prime}: \mathrm{H}^{3}\left(\mathfrak{X}_{\mathbb{Z}_{p}}, s_{\mathbb{Q}_{p}}^{\log }(2)\right) \rightarrow \mathrm{H}^{1}\left(\mathbb{Q}_{p}, V\right)
$$

The result we need is the following inclusion [17, Lemma 2.12] (which by [17], and more generally [19, Theorem 3.1], is in fact known to be an equality)

$$
\operatorname{im}\left(\eta^{\prime}\right) \subseteq \mathrm{H}_{g}^{1}\left(\mathbb{Q}_{p}, V\right)
$$

In particular, by $(10),(12)$ and (13) the image of $\mathrm{NH}_{\text {ét }}^{3}\left(\mathfrak{X}, \mathbb{Q}_{p}(2)\right)$ in $\mathrm{H}^{1}\left(\mathbb{Q}_{p}\right.$, $V$ ) lies in the subspace $\mathrm{H}_{g}^{1}\left(\mathbb{Q}_{p}, V\right)$, thus (8) induces a surjective map

$$
\mathrm{H}_{\text {ét }}^{3}\left(\mathfrak{X}, \mathbb{Q}_{p}(2)\right) / \mathrm{N} \mathrm{H}_{\text {èt }}^{3}\left(\mathfrak{X}, \mathbb{Q}_{p}(2)\right) \rightarrow \mathrm{H}^{1}\left(\mathbb{Q}_{p}, V\right) / \mathrm{H}_{g}^{1}\left(\mathbb{Q}_{p}, V\right)=: \Theta_{p}
$$

It remains to show $\Theta_{p} \neq 0$. The dimension of this space can be computed as follows [15, pg. 16] (see also [16, proof of Lemma 4-5]): Let $W=V(1)^{*} \cong$ $\mathrm{H}_{\text {ét }}^{2}\left(\bar{X}, \mathbb{Q}_{p}(1)\right)$, viewed as a $G_{p}=\operatorname{Gal}\left(\overline{\mathbb{Q}}_{p} / \mathbb{Q}_{p}\right)$-module. Then

$$
\operatorname{dim}_{\mathbb{Q}_{p}} \Theta_{p}=\operatorname{dim}_{\mathbb{Q}_{p}} D_{d R}(W) / D_{d R}^{0}(W)-\operatorname{dim}_{\mathbb{Q}_{p}} D_{c r i s}(W)^{f=1}+\operatorname{dim}_{\mathbb{Q}_{p}} W^{G_{p}} .
$$

For the first term we have isomorphisms

$$
\mathrm{D}_{d R}(W)=\mathrm{H}_{d R}^{2}(X / \mathbb{Q}) \otimes \mathbb{Q}_{p} \text { and } D_{d R}^{0}(W)=\mathrm{F}_{\mathrm{Hdg}}^{1} \mathrm{H}_{d R}^{2}(X / \mathbb{Q}) \otimes \mathbb{Q}_{p},
$$

hence the dimension of this term is equal to $\operatorname{dim} \mathrm{H}^{2}\left(X, \mathcal{O}_{X}\right)=1$.

For the second term, let $E_{\mathbb{F}_{p}}$ be the reduction of $E$ at the prime $p$, and let $X_{\mathbb{F}_{p}}=E_{\mathbb{F}_{p}} \times E_{\mathbb{F}_{p}}$. By the crystalline conjecture (cp. [9] and [6]) and the 
crystalline Tate conjecture (proved for abelian varieties in [21] and [13])

$$
D_{\text {cris }}(W)^{f=1} \cong\left(\mathrm{H}_{\text {cris }}^{2}\left(X_{\mathbb{F}_{p}} / W\left(\mathbb{F}_{p}\right)\right) \otimes \mathbb{Q}_{p}\right)^{\phi_{p}=p} \cong \operatorname{Pic}\left(X_{\mathbb{F}_{p}}\right) \otimes \mathbb{Q}_{p},
$$

and therefore

$$
\operatorname{dim}_{\mathbb{Q}_{p}} \mathrm{D}_{\text {cris }}(W)^{f=1}=2+\operatorname{dim}_{\mathbb{Q}}\left(\operatorname{End}_{\mathbb{F}_{p}}\left(E_{\mathbb{F}_{p}}\right) \otimes \mathbb{Q}\right)=4 .
$$

For the remaining term, let $\mathrm{V}_{p}(E)=\mathrm{H}^{1}\left(\bar{E}, \mathbb{Q}_{p}(1)\right)$, so that

$$
\operatorname{dim}_{\mathbb{Q}_{p}} W^{G_{p}}=2+\operatorname{dim}_{\mathbb{Q}_{p}} \operatorname{End}_{G_{p}}\left(\mathrm{~V}_{p}(E)\right)
$$

Here we use that we have ordinary reduction at $p$. It follows that the prime $p$ splits in the CM field $K, \mathbb{Q}_{p}$ contains $K$, and the complex multiplication is defined over $\mathbb{Q}_{p}$. Thus $\operatorname{dim}_{\mathbb{Q}_{p}} \operatorname{End}_{G_{p}}\left(V_{p}(E)\right)=2$, and therefore

$$
\operatorname{dim}_{\mathbb{Q}_{p}} \Theta_{p}=1-4+4=1
$$

Now the combination of (6), (14) and (15) implies that $\mathrm{CH}_{\mathrm{L}}^{2}(\mathfrak{X})\{p\}$ has positive corank, and completes the proof of part (a) of Theorem 1.1.

To prove $\mathrm{CH}_{\mathrm{L}}^{2}(X)\{p\}$ contains a copy of $\mathbb{Q}_{p} / \mathbb{Z}_{p}$ we show that the kernel of the map

$$
\mathrm{CH}_{\mathrm{L}}^{2}(\mathfrak{X})\{p\} \rightarrow \mathrm{CH}_{\mathrm{L}}^{2}(X)\{p\}
$$

is finite. Consider the commutative diagram with exact rows and columns

$$
\begin{aligned}
& \bigoplus_{v \notin S} \mathrm{H}_{\text {ét }}^{1}\left(Y_{v}, \mathbb{Q}_{p} / \mathbb{Z}_{p}(1)\right) \\
& \downarrow \\
& 0 \rightarrow \mathrm{H}_{\mathrm{L}}^{3}(\mathfrak{X}, \mathbb{Z}(2)) \otimes \mathbb{Q}_{p} / \mathbb{Z}_{p} \rightarrow \mathrm{H}_{\text {ét }}^{3}\left(\mathfrak{X}, \mathbb{Q}_{p} / \mathbb{Z}_{p}(2)\right) \quad \rightarrow \mathrm{CH}_{\mathrm{L}}^{2}(\mathfrak{X})\{p\} \rightarrow 0 \\
& \alpha \downarrow \quad \beta \downarrow \downarrow \gamma \\
& 0 \rightarrow \mathrm{H}_{\mathrm{L}}^{3}(X, \mathbb{Z}(2)) \otimes \mathbb{Q}_{p} / \mathbb{Z}_{p} \rightarrow \mathrm{H}_{\text {ét }}^{3}\left(X, \mathbb{Q}_{p} / \mathbb{Z}_{p}(2)\right) \quad \rightarrow \mathrm{CH}_{\mathrm{L}}^{2}(X)\{p\} \rightarrow 0 \\
& \underset{v \notin S}{\oplus} \mathrm{H}_{\text {ét }}^{2}\left(Y_{v}, \mathbb{Q}_{p} / \mathbb{Z}_{p}(1)\right)
\end{aligned}
$$

whose middle column is the localization sequence in étale cohomology. We will make again use of Mildenhall's result [18, Theorem 5.8] that $\Sigma=$ $\operatorname{ker}\left\{\mathrm{CH}^{2}(\mathfrak{X}) \rightarrow \mathrm{CH}^{2}(X)\right\}$ is a finite group. Since in bidegree $(3,2)$ we can identify motivic and Lichtenbaum cohomology for both $\mathfrak{X}$ and $X$, we may 
rewrite the localization sequence in motivic cohomology with integral coefficients in the form

$$
\mathrm{H}_{\mathrm{L}}^{3}(\mathfrak{X}, \mathbb{Z}(2)) \rightarrow \mathrm{H}_{\mathrm{L}}^{3}(X, \mathbb{Z}(2)) \stackrel{\partial}{\rightarrow} \underset{v \notin S}{\oplus} \mathrm{CH}^{1}\left(Y_{v}\right) \rightarrow \Sigma \rightarrow 0 .
$$

From the map 'multiplication by $p^{r}$ ' we obtain a long exact sequence

$$
\Sigma\left[p^{r}\right] \rightarrow \operatorname{im}(\partial) / p^{r} \rightarrow \underset{v \notin S}{\oplus} \mathrm{CH}^{1}\left(Y_{v}\right) / p^{r} \rightarrow \Sigma / p^{r} \rightarrow 0,
$$

and taking the direct limit over all $r$ it is immediate that the map

$$
\operatorname{coker}(\alpha)=\operatorname{im}(\partial) \otimes \mathbb{Q}_{p} / \mathbb{Z}_{p} \rightarrow \underset{v \notin S}{\oplus} \mathrm{CH}^{1}\left(Y_{v}\right) \otimes \mathbb{Q}_{p} / \mathbb{Z}_{p}
$$

has finite kernel. If $Y_{v}$ is a smooth closed fiber, we have from the Kummer sequence an injective map $\mathrm{CH}^{1}\left(Y_{v}\right) \otimes \mathbb{Q}_{p} / \mathbb{Z}_{p} \rightarrow \mathrm{H}_{\text {ét }}^{2}\left(Y_{v}, \mathbb{Q}_{p} / \mathbb{Z}_{p}(1)\right)$ such that the diagram

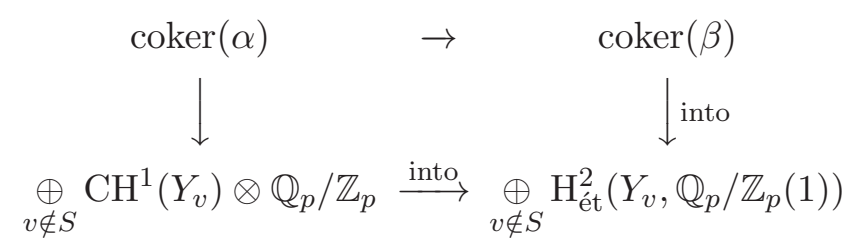

commutes. Thus the kernel of the top horizontal map is finite. Given this, it suffices to show the image of $\operatorname{ker}(\beta) \rightarrow \operatorname{ker}(\gamma)$, or equivalently, the image of

$$
\underset{v \notin S}{\oplus} \mathrm{CH}_{\mathrm{L}}^{1}\left(Y_{v}\right)\{p\} \rightarrow \mathrm{CH}_{\mathrm{L}}^{2}(\mathfrak{X})\{p\}
$$

is finite, which also follows easily from Mildenhall's Theorem. The diagram

$$
\begin{aligned}
\underset{v \notin S}{\oplus} \mathrm{CH}^{1}\left(Y_{v}\right)\{p\} & \rightarrow \mathrm{CH}^{2}(\mathfrak{X})\{p\} \\
& \cong \downarrow \\
\underset{v \notin S}{\oplus} \mathrm{CH}_{\mathrm{L}}^{1}\left(Y_{v}\right)\{p\} & \rightarrow \mathrm{CH}_{\mathrm{L}}^{2}(\mathfrak{X})\{p\}
\end{aligned}
$$

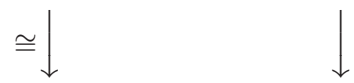

commutes, and the top horizontal map factors through the finite group $\Sigma\{p\}$. This proves our second claim (b), and completes the proof of Theorem 1.1.

Remark 3.1. If $E / \mathbb{Q}$ does not have complex multiplication and $p>3$ is a prime such that $E$ has good ordinary reduction at $p$, it follows from the proof of [16, Lemma 4-5] that $\operatorname{dim}_{\mathbb{Q}_{p}} \Theta_{p}=0$. 
We prove Corollary 1.2.

Proof. (of Corollary 1.2) It suffices to show that there is an exact sequence

$$
\mathrm{H}_{\mathrm{M}}^{3}\left(\mathfrak{X}, \mathbb{Q}_{p} / \mathbb{Z}_{p}(2)\right) \stackrel{\kappa}{\rightarrow} \mathrm{H}_{\text {ét }}^{3}\left(\mathfrak{X}, \mathbb{Q}_{p} / \mathbb{Z}_{p}(2)\right) \rightarrow \mathrm{H}_{\mathrm{nr}}^{3}\left(\mathfrak{X}, \mathbb{Q}_{p} / \mathbb{Z}_{p}(2)\right) .
$$

Given this, we have using (A) a commutative diagram with exact rows

$$
\begin{aligned}
0 & \rightarrow \mathrm{H}_{\mathrm{M}}^{3}(\mathfrak{X}, \mathbb{Z}(2)) \otimes \mathbb{Q}_{p} / \mathbb{Z}_{p} \rightarrow \mathrm{H}_{\mathrm{M}}^{3}\left(\mathfrak{X}, \mathbb{Q}_{p} / \mathbb{Z}_{p}(2)\right) \rightarrow \mathrm{CH}^{2}(\mathfrak{X})\{p\} \rightarrow 0 \\
& \cong \downarrow \\
0 & \rightarrow \mathrm{H}_{\mathrm{L}}^{3}(\mathfrak{X}, \mathbb{Z}(2)) \otimes \mathbb{Q}_{p} / \mathbb{Z}_{p} \rightarrow \mathrm{H}_{\text {ét }}^{3}\left(\mathfrak{X}, \mathbb{Q}_{p} / \mathbb{Z}_{p}(2)\right) \rightarrow \mathrm{CH}_{\mathrm{L}}^{2}(\mathfrak{X})\{p\} \rightarrow 0
\end{aligned}
$$

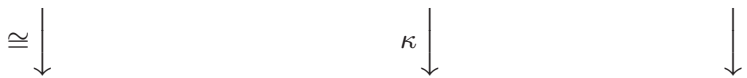

and Corollary 1.2 follows from Theorem 1.1. To establish (16) we first work with finite coefficients. Consider the spectral sequence (cp. [11, §4])

$$
\mathrm{E}_{1, \mathrm{M}}^{a, b}=\underset{x \in \mathfrak{X}^{(a)}}{\bigoplus} \mathrm{H}_{\mathrm{M}}^{b-a}\left(\kappa(x), \mathbb{Z} / p^{r} \mathbb{Z}(2-a)\right) \Rightarrow \mathrm{H}_{\mathrm{M}}^{a+b}\left(\mathfrak{X}, \mathbb{Z} / p^{r} \mathbb{Z}(2)\right),
$$

where $\mathrm{E}_{1, \mathrm{M}}^{a, b}=0$ for $a>2$, since $\mathbb{Z} / p^{r} \mathbb{Z}(n)=0$ for $n<0$. Moreover, $\mathrm{E}_{2, \mathrm{M}}^{0,3} \subseteq$ $\mathrm{H}^{0}\left(X, \mathcal{H}_{M}^{3}\left(\mathbb{Z} / p^{r} \mathbb{Z}(2)\right)\right)=0$, which implies that there is a surjective map

$$
\mathrm{H}_{\mathrm{M}}^{3}\left(\mathfrak{X}, \mathbb{Z} / p^{r} \mathbb{Z}(2)\right) \rightarrow \mathrm{E}_{2, \mathrm{M}}^{1,2}
$$

By construction, the group $\mathrm{E}_{2, \mathrm{M}}^{1,2}$ coincides with the homology of the complex

$$
\mathrm{K}_{2}(K) / p^{r} \rightarrow \underset{x \in \mathfrak{X}^{(1)}}{\bigoplus} \kappa(x)^{\times} / p^{r} \rightarrow \underset{x \in \mathfrak{X}^{(2)}}{\bigoplus} \mathbb{Z} / p^{r} \mathbb{Z}
$$

here we have used that $\mathrm{K}_{i}(F) / p^{r} \cong \mathrm{H}_{\mathrm{M}}^{i}\left(F, \mathbb{Z} / p^{r} \mathbb{Z}(i)\right)$ for a field $F$ and $i=$ $0,1,2$. There is an analogous spectral sequence for étale cohomology

$$
\mathrm{E}_{1, \text { ét }}^{a, b}=\underset{x \in \mathfrak{X}^{(a)}}{\bigoplus} \mathrm{H}_{\text {ét }}^{b-a}\left(\kappa(x), \mu_{p^{r}}^{\otimes(2-a)}\right) \Rightarrow \mathrm{H}_{\text {ét }}^{a+b}\left(\mathfrak{X}, \mu_{p^{r}}^{\otimes 2}\right) .
$$

In this spectral sequence $\mathrm{E}_{1 \text {,ét }}^{a, b}=0$ if $a>b$, since étale cohomology vanishes in negative degrees. Note $\mathrm{E}_{2 \text {,ét }}^{0,3}=\mathrm{H}_{\mathrm{nr}}^{3}\left(\mathfrak{X}, \mu_{p^{r}}^{\otimes 2}\right)$, thus there is an exact sequence

$$
0 \rightarrow \mathrm{E}_{2, \text { ét }}^{1,2} \rightarrow \mathrm{H}_{\text {ét }}^{3}\left(\mathfrak{X}, \mu_{p^{r}}^{\otimes 2}\right) \rightarrow \mathrm{H}_{\mathrm{nr}}^{3}\left(\mathfrak{X}, \mu_{p^{r}}^{\otimes 2}\right),
$$

where $\mathrm{E}_{2, \text { ét }}^{1,2}$ is the homology of the complex

$$
\mathrm{H}_{\text {ét }}^{2}\left(K, \mu_{p^{r}}^{\otimes 2}\right) \rightarrow \underset{x \in \mathfrak{X}^{(1)}}{\bigoplus} \mathrm{H}_{\text {ét }}^{1}\left(\kappa(x), \mu_{p^{r}}^{\otimes 1}\right) \rightarrow \underset{x \in \mathfrak{X}^{(2)}}{\bigoplus} \mathrm{H}^{0}\left(\kappa(x), \mu_{p^{r}}^{\otimes 0}\right)
$$


Now the complexes (18) and (20) are isomorphic, since $K_{i}(F) / p^{r} \cong \mathrm{H}_{\text {ét }}^{i}(F$, $\left.\mu_{p^{r}}^{\otimes i}\right)$ for a field $F$ and $i=0,1,2$. Thus $\mathrm{E}_{2, \mathrm{M}}^{1,2} \cong \mathrm{E}_{2, \text { ét }}^{1,2}$, and we obtain from (17) and (19) the exact sequence $\mathrm{H}_{\mathrm{M}}^{3}\left(\mathfrak{X}, \mathbb{Z} / p^{r} \mathbb{Z}(2)\right) \rightarrow \mathrm{H}_{\text {ét }}^{3}\left(\mathfrak{X}, \mu_{p^{r}}^{\otimes 2}\right) \rightarrow \mathrm{H}_{\mathrm{nr}}^{3}\left(\mathfrak{X}, \mu_{p^{r}}^{\otimes 2}\right)$; given this, the exact sequence (16) follows by taking the evident colimit.

For the second statement of the Corollary note that there is an inclusion

$$
\mathrm{H}_{\mathrm{nr}}^{3}\left(\mathfrak{X}, \mathbb{Q}_{p} / \mathbb{Z}_{p}(2)\right) \subseteq \mathrm{H}_{\mathrm{nr}}^{3}\left(X, \mathbb{Q}_{p} / \mathbb{Z}_{p}(2)\right)
$$

Remark 3.2. Let $X$ be a smooth quasi-projective variety over a field $k$. From [12, Proposition 2.9] we have the exact sequence

$$
0 \rightarrow \mathrm{CH}^{2}(X) \rightarrow \mathrm{CH}_{\mathrm{L}}^{2}(X) \rightarrow \mathrm{H}_{\mathrm{nr}}^{3}(X, \mathbb{Q} / \mathbb{Z}(2)) \rightarrow 0,
$$

and Theorem 1.1 also implies that for the surface $X / \mathbb{Q}$ the groups $\mathrm{CH}^{2}(X)$ and $\mathrm{CH}_{\mathrm{L}}^{2}(X)$ differ by an infinite group; however, if $\bar{X}=X \times_{\mathbb{Q}} \overline{\mathbb{Q}}$, then $\mathrm{CH}^{2}(\bar{X})=\mathrm{CH}_{\mathrm{L}}^{2}(\bar{X})$.

\section{Acknowledgements}

The first author gratefully acknowledges support by the DFG. The second author was supported during this work by the Humboldt foundation and a J. C. Bose Fellowship of the Department of Science and Technology, India.

\section{References}

[1] Spencer Bloch, Algebraic K-theory and classfield theory for arithmetic surfaces. Ann. of Math. (2), 114, (1981), 229-265.

[2] Spencer Bloch, Algebraic cycles and higher K-theory. Adv. in Math., 61, (1986), 267-304.

[3] Spencer Bloch and Kazuya Kato, L-functions and Tamagawa numbers of motives. In: The Grothendieck Festschrift, Vol. I, Progr. Math., 86, 333-400, Birkhäuser Boston, Boston, MA, 1990.

[4] Armand Brumer, Galois groups of extensions of number fields with given ramification. Michigan Math. J., 13, (1966), 33-40.

[5] J.-L. Colliot-Thélène and W. Raskind, $\mathcal{K}_{2}$-cohomology and the second Chow group. Math. Ann., 105, (1985), 165-199. 
[6] Gerd Faltings, Crystalline cohomology and p-adic Galoisrepresentations. In: Algebraic analysis, geometry, and number theory (Baltimore, MD, 1988), 25-80, Johns Hopkins Univ. Press, Baltimore, MD, (1989).

[7] Matthias Flach, Selmer groups for the symmetric square of an elliptic curve. Thesis, Cambridge University, (1990).

[8] Jean-Marc Fontaine, Sur certains types de représentations p-adiques du groupe de Galois d'un corps local; construction d'un anneau de BarsottiTate. Ann. of Math. (2), 115, (1982), 529-577.

[9] Jean-Marc Fontaine and William Messing, p-adic periods and p-adic étale cohomology. In: Current trends in arithmetical algebraic geometry (Arcata, Calif., 1985), Contemp. Math., 67, 179-207, Amer. Math. Soc., Providence, RI, (1987).

[10] Thomas Geisser and Marc Levine, The Bloch-Kato conjecture and a theorem of Suslin-Voevodsky. J. Reine Angew. Math., 530, (2001), 55103.

[11] Thomas Geisser, Motivic cohomology over Dedekind rings. Math. Z., 248, (2004), 773-794.

[12] Bruno Kahn, Classes de cycles motiviques étales. Algebra Number Theory, 6, (2012), 1369-1407. 
[13] Nicholas M. Katz and William Messing, Some consequences of the Riemann hypothesis for varieties over finite fields. Invent. Math., 23, (1974), 73-77.

[14] Kazuya Kato, Semi-stable reduction and p-adic étale cohomology. In: Périodes p-adiques (Bures-sur-Yvette, 1988), Astérisque, 223, (1994), 269-293.

[15] Andreas Langer and Wayne Raskind, 0-cycles on the self-product of a CM elliptic curve over Q. J. Reine Angew. Math., 516, (1999), 1-26.

[16] Andreas Langer and Shuji Saito, Torsion zero-cycles on the self-product of a modular elliptic curve. Duke Math. J., 85, (1996), 315-357.

[17] Andreas Langer, Local points of motives in semistable reduction. Compositio Math., 116, (1999), 189-217.

[18] Stephen J. M. Mildenhall, Cycles in a product of elliptic curves, and a group analogous to the class group. Duke Math. J., 67, (1992), 387-406.

[19] Jan Nekovář, p-adic Abel-Jacobi maps and p-adic heights. In: The arithmetic and geometry of algebraic cycles (Banff, AB, 1998), CRM Proc. Lecture Notes, 24, 367-379, Amer. Math. Soc., Providence, RI, (2000).

[20] Shuji Saito and Kanetomo Sato, A p-adic regulator map and finiteness results for arithmetic schemes. Doc. Math., Extra volume, Andrei A. Suslin sixtieth birthday, (2010), 525-594.

[21] John Tate, Endomorphisms of abelian varieties over finite fields. Invent. Math., 2, (1966), 134-144.

[22] Takeshi Tsuji, On p-adic nearby cycles of log smooth families. Bull. Soc. Math. France, 128, (2000), 529-575.

[23] Vladimir Voevodsky, Motivic cohomology with Z/2-coefficients. Publ. Math. Inst. Hautes Études Sci., 98, (2003), 59-104.

[24] Vladimir Voevodsky, On motivic cohomology with $\mathbf{Z} /$ l-coefficients. Ann. of Math., 174, (2011), 401-438.

[25] C. Weibel, The norm residue isomorphism theorem. J. Topol., 2, (2009), 346-372. 
Mathematisches Institut, Ludwig-Maximilians-Universität

MünChen, Germany

E-mail address: axr@math.lmu.de

School of Mathematics, Tata Institute of Fundamental Research Mumbai, India

E-mail address: srinivas@math.tifr.res.in

Received November 3, 2014 
